\title{
Design and Fabrication of a Low Cost Implantable Bladder Pressure Monitor
}

\author{
F. Axisa ${ }^{1}$, P. Jourand ${ }^{2}$, E. Lippens ${ }^{3}$, M. Rymarczyk-Machal ${ }^{4}$, N. De Smet ${ }^{4}$, E. Schacht ${ }^{4}$, \\ J. Vanfleteren ${ }^{1}$, R. Puers ${ }^{2}$, R. Cornelissen ${ }^{3}$, \\ 1 CMST-University of Ghent/IMEC, Belgium \\ 2 ESAT-MICAS, Katholieke Universiteit Leuven, Belgium \\ 3 Histology group, University of Ghent, Belgium \\ 4 PBM, University of Ghent, Belgium
}

\begin{abstract}
In the frame of the Flemish Community funded project Bioflex we developed and fabricated an implant for short term bladder pressure monitoring, and diagnosis of incontinence. This implant is soft and flexible to prevent damaging the bladder's inner wall, and designed for a 7 days long implantation. It is based on a standard flexible electronic circuit connected to a battery, embedded in silicone, of which the surface is treated to enhance the biocompatibility and to prevent salt deposition. This article describes the fabrication of the pill. The implantation and the result of the in-vivo experimentation will be presented in another article.
\end{abstract}

\section{INTRODUCTION}

$\mathrm{I}$ $\mathrm{n}$ the frame of the Flemish Community funded project called Bioflex [4], research has been focused on the development of biocompatible, flexible or stretchable, electronic systems for implantation, based on PDMS substrates. This project is based on a consortium of universities and research groups. The MICAS group of the Katholieke Universiteit Leuven is responsible for designing electronic systems for biomedical applications. The CMST group of the University of Ghent is responsible for the technology development. The PBM group of the University of Ghent is responsible for the material development, and especially of the PDMS surface modification. The Histology group of the University of Ghent is responsible for the in vitro cytotoxicity tests and in vivo experimentations. The Bioflex consortium is also working with the faculty of veterinary medicine of Ghent for in-vivo implantation.

This article describes the development of a low cost flexible implantable pill for short term ( 7 days) pressure monitoring inside the bladder. Development of stretchable electronic has been described in several articles [3,5]. Moreover, this article is a short presentation of the implantable pill. Each part of the process, and detailed results and description will be published afterwards.

The development of the implantable pill is done in four phases: electronic system development and tests of functionality, fabrication and encapsulation in PDMS of the implant, surface modification of the pill, and toxicology test.

\section{Methodology}

In order to monitor during a short term (7days) the pressure inside the bladder for diagnostic purposes of urinal or nephrological disorders [6], the idea is to install a pill shaped implant inside, to record the pressure during one week, and then to recuperate the pill using natural way, for data analysis by the doctor[1]. We want to develop a low cost implant, using standard and available technologies, biocompatible enough to be inside a bladder during 7 days, and as soft as possible. As the bladder is a very stretchable organ, the implant should be soft and conformable enough not to harm the bladder inner wall when its volume is minimal, and to float when the bladder is filling with urine.

The implant is pill shaped in order to enable the introduction in the bladder with a catheter. This constraint forces us to have very thigh geometrical constraints, in order to fit as many functions as possible within a maximum diameter of $5 \mathrm{~mm}$. The length of the pill is not as critical, however the smaller the better. As a trade-off between implement ability and need-for-space, a length of $40 \mathrm{~mm}$ has been defined. Different shapes of the implant have been tested (pill shape, pill with flaps to extend the surface of the pill, pill with whiskers to avoid undesirable evacuation), however the functional implant is a pill shape, as it is the easiest to implant using a catheter.

Two solutions for electronic systems were possible. In the first solution, the pill has no battery, and it is powered using an inductive link. This inductive link is also used for communication [2]. However the position and the orientation of the implant in the bladder are always changing. So in order to have a correct coupling between the implant and the outside a 3D inductive coupling should be set from the inside to the outside of the bladder, which is not a convenient solution for the patient, and is also volume consuming for the implant. The second solution is to integrate a battery and a memory into the implant. Therefore communication is not possible during the implantation; it is not possible to control the functionality or the data during experimentation. Moreover a battery is highly volume consuming, capacity is limited, and no flexible battery with enough capacity exists yet. A mixed solution which consists in a battery and a communication system is even more volume consuming, even though it would be a better solution in term of functionality.

The sensor is a key part of the device. The sensor should be protected from the fluids; however it should be able to sense the pressure. So the protection should be as minimal as possible. Therefore a window is created in the encapsulation, in order to cover the sensor with just a very thin PDMS 
membrane of $100 \mu \mathrm{m}$. Moreover, as urine is a very salty solution, salt deposition can happen on the surface of the PDMS, which can affect the mechanical properties of the membrane, affecting the measurements. A surface treatment, to enhance the biocompatibility of the PDMS and to prevent the deposition of salt on its surface is therefore needed.

\section{RESULT}

\section{A. Electronic Design}

The electronic design of this application was done taking into account the following rules: everything must fit into a $5 \mathrm{~mm}$ diameter capsule with a length of $40 \mathrm{~mm}$ including the battery; data must be stored on a memory module and read out after extraction of the implant and the power from the battery must be able to be switched off after encapsulation. The block diagram of the bladder pill is depicted in Figure 1. The device uses a surface machined capacitive absolute pressure sensor from MicroFab (E1.3N). Its range (0.5 to $1.3 \mathrm{bar}$ ) and size are perfectly suited for the application. This signal is digitized by a capacitance to digital converter from Analog Devices (AD7153). The CDC is a dedicated IC configured and controlled through $\mathrm{I}^{2} \mathrm{C}$. To conserve as much power as possible, it is set to single conversion mode in a range of $0.25 \mathrm{pF}$.

The backbone is a microcontroller unit (MCU) adding intelligence to the device. The choice in MCU involves an important tradeoff: the physical size must me as small as possible, yet this comes with limited functionalities and programming memory. The PIC10F206 from Microchip was selected mainly for its tiny SOT23 package. Its sole useful (for this application that is) functionalities are: an on board clock oscillator and an 8 bit timer. All other needed functionalities: time tracking, configuration of the $\mathrm{CDC}$ at startup, $\mathrm{I}^{2} \mathrm{C}$ communication and the processing and storing of the results, are implemented in its $512 \mathrm{k}$ program memory. The MCU will fetch a CDC result every $500 \mathrm{~ms}$. Data will eventually be stored in an EEPROM memory of $16 \mathrm{Kbyte}$, which was selected for it also uses $\mathrm{I}^{2} \mathrm{C}$, and its miniature size in a 5pin SOT23 package. It is because of this limited memory the MCU must first process the results.

A data result of 16 bits will contain only 5 bits of pressure data and 11 bits of timing data. Focus was shifted from pressure accuracy to time because in this first stage, pressure measurements do not need to be accurate making sure that a lot of data can be stored. Since the expected bladder pressure remains the same for long periods, this would make sure that in an ideal case where $2^{11}$ consecutive pressure samples, covering $1024 \mathrm{~s}$ of the same pressure reading, will only take up 16 bits of data.

Power to all components comes indirectly from a $3 \mathrm{~mm}$ diameter pin-type lithium battery with a body of $13 \mathrm{~mm}$. Its capacity is a mere $13 \mathrm{mAh}$. To conserve power between production and implantation, a magnetic switch circuit is introduced with a reed switch and a D-Flip-Flop. A 0603size LED is also used to signal the on and off state of the device.
Both measuring and power switch circuitries were then fitted onto a double-sided flex sizing $4.2 \times 18 \mathrm{~mm} 2$. From this file, the component outlines were put into an AutoCAD file to help the production of the molds for the actual fabrication, which will be discussed later. This AutoCAD design is shown in Figure 2. It also gives a general idea on how the pill will look like and what the relative sizes of the different components are.

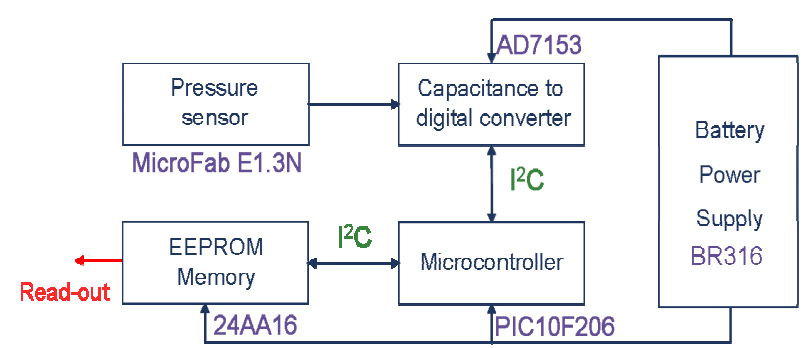

Figure 1 Block diagram of the bladder pill

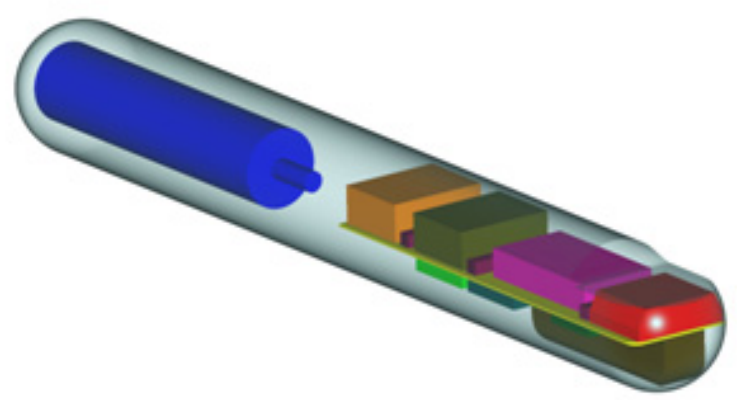

\section{Figure 2 3D-Model of the bladder pill showing the battery and circuitry in a pill measuring $65 \mathrm{~mm} \times 40 \mathrm{~mm}$}

\section{B. Programming}

The microcontroller is programmed before soldering on the electronic flexible substrate. When the implant is switched on, a LED is blinking and the microcontroller takes pressure measurements during 8 seconds, and then switches to sleep mode during 5 hours. Then it starts taking a pressure measurement once every 0.5 seconds until the memory is full or until the battery is empty. The data are then recovered after extraction of the pill by reading directly the EEPROM.

\section{Fabrication of the implant}

From the electronic design layout, the photolithographic masks are edited for the fabrication of the flex. At first, a polyimide substrate coated on both sides with copper (UPILEX, $25 \mu \mathrm{m}$ of polyimide, $9 \mu \mathrm{m}$ of copper on both sides) is laser drilled to define the vias and the alignment marks). Then, a superficial layer of copper is electroless plated to make the vias conductive. Then, copper is electroplated to obtain a total additional copper layer of 10 $\mu \mathrm{m}$ on both sides and in the vias. The following step is to process lithography on both sides, and copper is etched in $\mathrm{CuCl} 2$ based etchant. After stripping in $\mathrm{NaOH}$, solder mask ELPEMER SD 2463 Flex HD is screen printed and cured. Then electroless plating of $\mathrm{Ni} / \mathrm{Au}$ finish the surface of the mask. 
The second step in the fabrication is the soldering of the components. A lead free solder paste (Delphine, $\mathrm{SnAg}(3 \%) \mathrm{Cu}(0.5 \%))$ is dispensed on the pads, components are placed and soldering is done in a reflow oven at $270{ }^{\circ} \mathrm{C}$. After soldering the two sides, the flex is cut off the substrate using a YAG laser. Figure 3 shows the flexible substrate at the three stages: after lithography, after $\mathrm{Ni} / \mathrm{Au}$ finishing and after components soldering. After functionality testing, the flexes are ready to be encapsulated.

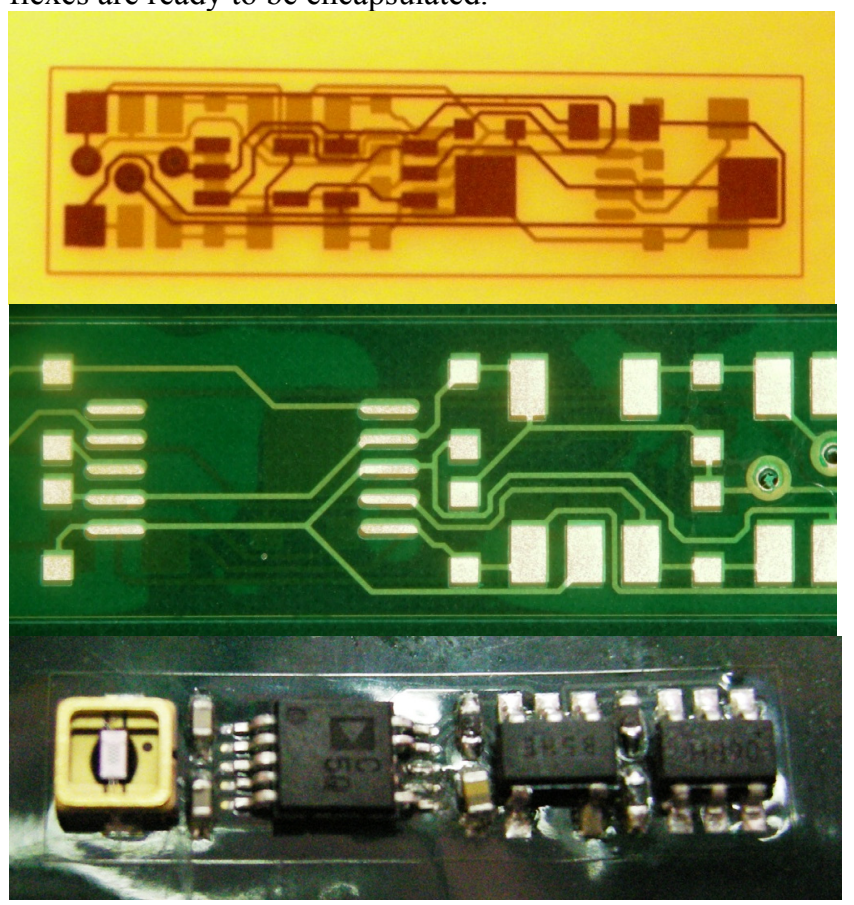

Figure 3 Fabrication of the flexible electronic substrate

From the 3D model (Figure 2), the injection mould for the external shape of the implant and of the Teflon holder to fixate the system during molding are designed in AutoCAD. The mould is fabricated from transparent PMMA, transparent enough to control the injection and to avoid any air bubble formation. The tool used to fabricate the mould is a digital milling machine. The measurement of the smoothness of mould is done with WYKO laser profilometer, the roughness of the mould is $13 \mu \mathrm{m}$. In order to fixate the flexible substrate and the battery during the molding, a Teflon holder is processed in the same way. The precision of the Teflon holder is crucial to obtain a correct positioning of the sensor in the pill. A precision of $50 \mu \mathrm{m}$ is obtained. Then tests to check the mould are performed, in order to control the thickness of the membrane on the top of the sensor. The thickness varies from $76 \mu \mathrm{m}$ to $170 \mu \mathrm{m}$. As there is no sufficiently small battery connector, we are using a copper filament with a $40 \mu \mathrm{m}$ diameter wrapped tidily around the battery and soldered with lead free solder. The battery is then soldered to the flex using these wires. The implant is put into switched-off state and then dipped in ethanol to clean, dried at room temperature during 1 hour, and then it is dipped in biocompatible adhesion promoter (NUSIL MED6-161). After 45 minutes, the adhesion promoter is hydrolyzed enough. Then the system (flex and battery) is glued on the holder with MDX 4210 Biocompatible silicone rubber from Dow Corning. The glue is cured at $40{ }^{\circ} \mathrm{C}$ during 4 hours to avoid any thermal deformation of the mould and to avoid overheating the battery. The top of the system is coated again with the adhesion promoter using the same process, and then the top mould is set on the holder and screwed tidily. Fully degassed MDX 4210 biocompatible PDMS is then injected using a syringe and a vacuum pomp to remove any entrapped air during the injection to avoid air bubbles. Then the PDMS is cured at room temperature during 24 hours. Afterwards, the holder is removed, and as the adhesion Silicone/PMMA is higher than the adhesion Silicone/Teflon, the molded system stays in the top mould. To enhance wet ability of the silicone, the molded system is plasma treated (low pressure air plasma, 0.8 mbar air, 197W, 30s). To enhance the adhesion silicone/silicone, the surface is coated with the biocompatible adhesion promoter using the same process. After sealing the bottom mould to the top mould, MDX 4210 is injected in the bottom mould. After 24hours of curing at room temperature, the pill is un-molded, the overflowed PDMS cut out with a sharp scalpel and the pill is cleaned in ethanol and its functionality is tested.

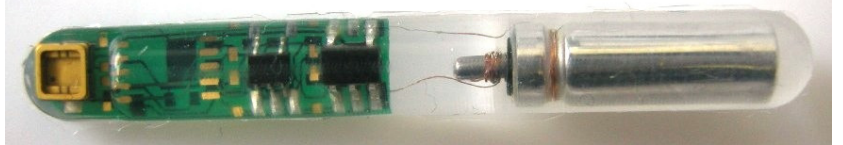

Figure 4 Implant after encapsulation in MDX 4210 PDMS

\section{PDMS surface modification}

In order to reduce the deposition of salts (encrustration problem) on the surface of the bladder implant, the device was surface modified by plasma induced grafting of acrylamidopropyl sulfonic acid (AMPS) or sulfobetaïne. The grafted surfaces were analysed by contact angle measurements, XPS and FT-IR surface imaging. In vitro and preliminary in vivo experiments have demonstrated that such surface modification significantly reduces the salt deposition.

\section{E. Toxicity test}

In order to control the biocompatibility of the implant (material+process), a cytotoxicity test of 8 days is performed. Cytotoxicity tests with the extraction products of the silicone materials in culture medium were performed in triplicate in order to evaluate the presence of toxic leachables and metals, following the ISO 10 993-1 guidelines. Samples were sterilized by ethylene oxide gas. The extraction took place in a tube with $2.5 \mathrm{ml}$ culture medium (Hank's medium with 10 vol\% Fetal bovine serum) at $37^{\circ} \mathrm{C}$ under dynamic conditions for 1 week. This medium was subsequently added to a subconfluent cell layer of primary derived chicken embryo fibroblasts. 24 hours later, the influence of the extraction products on the cell viability was evaluated with the MTT assay. The mitochondria of viable cells reduced this MTT tetrazolium salt into a formazan product. After dissolving the formazan in isopropanol $/ \mathrm{HCl}$ with $1 \%$ Triton $\mathrm{X} 100$, the absorbance could 
be determined spectrophotometrically $(\lambda=580 \mathrm{~nm})$. This absorbance value is proportional to the number of viable cells and can be compared to the absorbance value of control cell culture without contact with the extraction medium.

The protocol is: extraction in long cryoval $(2.5 \mathrm{ml}$ per pill), dynamic incubation at $37{ }^{\circ} \mathrm{C}$ during 1 week in Hanks+FCS incubation medium. Then extraction medium is used immediately to react with 7 days old chicken embryo fibroblasts in a well. Each well contains 40000 cells and the extraction test reactant. The extraction test reactant is MTT. MTT is based on tetrazolium salt which is reduced to formazan by the fibroblasts if they are alive. MTT turns from translucent to purple if the cells are alive. After 4 hours of incubation, a light absorbance test is performed to determine the percentage of cell survival. The absorbance of the formazan product is directly proportional to the number of viable cells in the culture.

The rate of viability is the ratio of the absorbance of the sample with the absorbance of a control sample. 6 samples of the pill implant were tested: 2 samples of a battery embedded in the silicone pill (Battery A and Battery B), 2 samples of a battery and flex embedded in the silicone pill (Flex A and Flex B) and 2 samples of a battery + flex + components embedded in the silicone pill (Components $\mathrm{A}$ and Components B) (Figure 5).

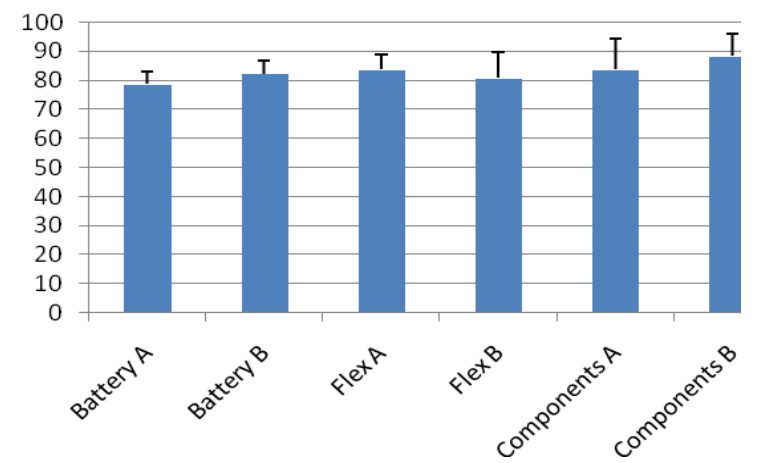

Figure 5 In Vitro biocompatibility tests of the implant

The vialibity of the implant for 7 days ranges from $78 \%$ to $98 \%$. Therefore it shows that the implant is not toxic for an implantation of 7 days in the bladder. Deeper analyses will be developped in forthcomings articles.

\section{DISCUSSION}

We have been able to produce a biocompatible, soft and flexible implant to measure the pressure in the bladder, using standard PCB technologies and PDMS encapsulation. Functionality has been tested as well. However, in-vivo experimentations and biological aspects of the implant have not been presented yet.

The production of this implant is low cost, as it uses only standard PCB technologies and commonly available components. The biocompatibility is limited to 7 days, as it is a diagnostic implant, and to reach this biocompatibility, the PDMS rubber MDX 4210 from Dow Corning is sufficient. However, post implantation biological analysis is needed to prove the biocompatibility of this implant. Biological analysis will have to prove the biochemical and biological compatibility, as well as the mechanical compatibility: As the bladder is a very elastic organ, the implant should not harm the inner wall.

\section{CONCLUSION}

This implant is a demonstration that low cost biocompatible implants can be produced for short term implantation for diagnostic purposes. Several improvements are needed: reducing the volume to ease the implantation using natural ways, improving the power management to increase the storage time and reduce the battery size, using bare chips instead of packaged chips to reduce the volume of the implant. However designing an implant for short term diagnostic purpose is a tradeoff between volume, cost and functionality.

\section{ACKNOWLEDGMENT}

This research has been developed in the frame of the research program BIOFLEX, an IWT funded project in the frame of the SBO (Strategic Basic Research), contract number IWT-040101.

\section{REFERENCES}

[1] J. Coosemans and R.Puers, "An autonomous Bladder Pressure Monitoring System" in Sensors and Actuator A: Physicals, vol. 123124,2005 , pp. $155-161$.

[2] J. Coosemans "Wireless and Battery -less medical Monitoring Devices" PhD, 2008, Katholieke Universiteit Leuven.

[3] D. Brosteaux et al "Design and fabrication of elastic interconnections for stretchable electronic circuits" , IEEE Electron Device Letters, vol 28, no 7, pp 552-554

[4] BIOFLEX Biocompatible Flexible Electronic Circuits, IWT-SBO, IWT-040101

[5] F. Axisa et al "Biomedical stretchable systems using MID based stretchable electronics technology", Proc. $29^{\text {th }}$ Annual IEEE EMBC, Lyon (France), 2007, pp 5687-5690

[6] Koldewijn et al, "Bladder pressure sensors in an animal" in J. Urol., 1994 May, 151(5) : 1379-84

[7] A. J. Courly, St. Paul, J. R. Keogh, M. N. Maplewood, Ch. M. Hobot, T. Bay United States Patent, Patent number: 5278200, Date of Patent Jan. 11, Thromboresistant material and articles (1994). 